\title{
Universal screening for SARS-CoV-2 in pregnant women should be weighed by population incidence of the disease
}

\author{
Elisabeth van Leeuwen ${ }^{1}$, Pim Teunissen ${ }^{2}$, Eva Pajkrt ${ }^{3}$, Janke Schinkel ${ }^{3}$, Christianne de \\ Groot $^{2}$, and Wessel Ganzevoort ${ }^{4}$ \\ ${ }^{1}$ Amsterdam University Medical Centres \\ ${ }^{2}$ Amsterdam UMC \\ ${ }^{3}$ Amsterdam Universitair Medische Centra \\ ${ }^{4}$ Amsterdam UMC - Locatie AMC
}

July 16, 2020

\begin{abstract}
Objectives: To evaluate universal SARS-CoV-2 screening in labouring women in a tertiary hospital in the Netherlands. Women with an unknown SARS-CoV-2 were treated as COVID-19 positive in theatre. As COVID-19 precautions differed from standard care, this may have contributed to adverse perinatal outcomes. Methods: Women admitted to the labour- and pregnancy ward were consecutively asked for COVID-19 symptoms and then screened for SARS-CoV-2 by PCR. Results: From March 52020 to May 13, 283 women without COVID-19 symptoms were screened. One post-symptomatic woman was excluded from the analysis. 3/ 282 women (1.1\%) tested positive for SARS-CoV-2. In one woman, a pending SARS-CoV-2 test, may have worsened perinatal asphyxia in her child, and in two other cases with post-partum haemorrhage the prolonged decision-to intervention interval may have contributed to more blood loss. Conclusion: Caretakers should balance the potential additional perinatal risks of alternative care processes for unscreened SARS-CoV-2 patients in obstetric emergencies.
\end{abstract}

\section{Introduction}

The role of presymptomatic (SARS-CoV-2 detected before symptom onset) or asymptomatic (SARS-CoV-2 detected but symptoms never develop) persons in the COVID-19 pandemic is still under debate [1]. As a result, symptom-based screening may not be sufficient and PCR testing is often recommended. The American Society of Anaesthesiologists recommends universal screening for SARS-CoV-2 by PCR in individuals scheduled for non-emergency surgery when SARS-CoV-2 prevalence is high, but advices on a symptom-based strategy when prevalence is low. The rationale is threefold; first, to protect personnel during aerosolizing airway management procedures, second to prevent complications after surgery [2]; and, third, to minimize SARS-CoV-2 spread to patients in recovery. Previous studies in pre-or asymptomatic women from the USA reported a SARS-CoV-2 prevalence ranging from $2.9 \%$ to $13.5 \%$ [3 4]. As labouring women are all at risk to undergo surgery, Amsterdam University Medical Centers (AUMC) has installed universal screening for SARS-CoV-2 of all women admitted to our labour- and pregnancy ward since the pandemic reached the Netherlands. Women with a positive or unknown SARS-CoV-2 are treated as COVID-19 positive if they are seen in theatre. This safety measure may lead to an increased decision-to-intervention time, because of COVID-19 precautions that differ from standard care, such as an alternative routing in theatre and a potential delay in preparing for incision. The rationale behind this approach should be weighed by population incidence of the disease. We aimed to evaluate this rationale in our setting.

\section{Methods}


All women were systematically asked for COVID-19 symptoms on admittance to the labour- and pregnancy ward of the AUMC, and were then screened consecutively for SARS-CoV-2 by a PCR on a throat- and/or nasopharyngeal swab [5]. If the PCR result was $>48$ hours old, the PCR was repeated. The Medical Ethics committee of the AUMC declared this study exempt from their evaluation (W20_257).

\section{Results}

From March 52020 to May 13, 283 women had no current COVID-19 symptoms and were screened for SARSCoV-2 by PCR. In total 335 tests were performed in 283 women (table 1). One woman who reported having symptoms suggestive for COVID-19 four weeks before was excluded from the analysis. Three women tested positive (1.1\%) out of the 282 other women. When questioned further, two suffered from mild symptoms; one had a sore throat, but she had not mentioned this out of fear of being refused treatment, one had an obstructed nose, and one was truly asymptomatic. In one woman, a pending SARS-CoV-2 test, and therefore a policy to adhere to COVID-19 protective measures during her emergency caesarean, contributed to a prolonged decision-to-intervention interval which in turn may have worsened perinatal asphyxia in her child. In another two cases with post-partum haemorrhage the prolonged decision-to intervention interval contributed to more pronounced blood loss.

\section{Discussion}

We here report a 1.1\% SARS-CoV-2 prevalence under presumed asymptomatic labouring and hospital admitted pregnant women, taken in a six weeks' interval that includes the peak incidence in Amsterdam in the end of March, beginning of April. These results are in contrast with other studies where percentages of SARS-CoV-2 in asymptomatic pregnant women were as high as 3-14\% [3 4]. Knowing that SARS-CoV-2 PCR testing will not have $100 \%$ specificity, there will be false positive test results. Consequently, in our setting PCR helped to increase a pre-test likelihood of not having COVID-19 of 98.9\% with an estimated 1 percentage point or less. It is therefore debatable which prevalence rates are high enough to justify a policy of universal PCR testing and full COVID-19 precautions if a patient has not been tested negative for SARS-CoV-2. Given the delay associated with personal protection equipment (PPE) for health care workers, and the potential for foetal damage in obstetric urgencies, these conflicting interests should be weighed carefully. Moreover, most pregnant women will undergo loco-regional anaesthesia rather than aerosolizing general anaesthesia. This further inflates the number of patients that needs to be screened to prevent viral transmission from a patient to a health care worker. Each hospital should therefore weigh the pros and cons of universal screening versus PPE in their own setting and background incidence, to balance the risks for health care workers versus the additional perinatal risk of alternative care processes for unscreened patients in obstetric emergencies.

\section{Conflicts of interests}

None of the authors have personal, political, intellectual or religious conflicts of interests or financial disclosures.

\section{Financial support}

None

\section{Ethical considerations}

The Medical Ethics committee of the AUMC declared this study exempt from their evaluation (number W20_257) and approved of publication of data. All patients agreed upon SARS-CoV-2 sampling.

\section{Contribution to authorship}

EvL and JWG wrote the manuscript.

JS, CdG and EvL provided and analysed data.

JS, PT, EP and CdG designed the commentary. 
JS provided virological expertise.

\section{References}

1. Nathan WF, John TB, Jeremy S. Evidence Supporting Transmission of Severe Acute Respiratory Syndrome Coronavirus 2 While Presymptomatic or Asymptomatic. Emerging Infectious Disease journal 2020;26 (7) doi: 10.3201/eid2607.201595[published Online First: Epub Date]|.

2. Aminian A, Safari S, Razeghian-Jahromi A, Ghorbani M, Delaney CP. COVID-19 Outbreak and Surgical Practice: Unexpected Fatality in Perioperative Period. Ann Surg 2020 doi: 10.1097/SLA.0000000000003925[published Online First: Epub Date]|.

3. Sutton D, Fuchs K, D'Alton M, Goffman D. Universal Screening for SARS-CoV-2 in Women Admitted for Delivery. N Engl J Med 2020;382 (22):2163-64 doi: 10.1056/NEJMc2009316[published Online First: Epub Date]|.

4. Campbell KH, Tornatore JM, Lawrence KE, et al. Prevalence of SARS-CoV-2 Among Patients Admitted for Childbirth in Southern Connecticut. JAMA 2020 doi: 10.1001/jama.2020.8904[published Online First: Epub Date]|.

5. Corman VM, Landt O, Kaiser M, et al. Detection of 2019 novel coronavirus (2019-nCoV) by real-time RT-PCR. Euro Surveill 2020;25 (3) doi: 10.2807/1560-7917.ES.2020.25.3.2000045[published Online First: Epub Date]|.

\begin{tabular}{lll}
\hline & $\mathrm{n}(\%)$ or median (IQR) & $\mathrm{n}(\%)$ or median (IQR) \\
\hline Total Number of women & 283 & \\
Pre- or asymptomatic & 282 & \\
Post symptomatic & 1 & $(1.1 \%)$ \\
SARS-CoV-2 positive & 3 & $(98,9 \%)$ \\
SARS-CoV-2 negative & 279 & \\
Total Number of PCR & 335 & $(36 \%)$ \\
PCR pregnancy WARD & 120 & $(64 \%)$ \\
PCR Labour & 215 & $(29-36)$ \\
Age & 32 & $(31-40)$ \\
Pregnancy duration & 38 & $(48 \%)$ \\
Nulliparous & 137 & \\
\hline
\end{tabular}

Table 1. Characteristics of asymptomatic pregnant women. 